\title{
First Report of Equine Leishmaniosis in the State of Sergipe, Brazil
}

\author{
Saulo Romero Felix Gonçalves', Mateus de Melo Lima Waterloo', Mariana Lumack de Monte Barretto', \\ João Paulo Gonçalves Moura'2, Andrea Alice da Fonseca Oliveira', \\ Jacilene Lourenço da Silva' \& Valdemiro Amaro da Silva Junior'
}

\begin{abstract}
Background: Leishmaniasis is caused by an obligate intracellular protozoan of the genus Leishmania. In Brazil, the species Leishmania (Leishmania) infantum is reported as an etiological agent of leishmaniasis. Transmission of the disease occurs through bite of the sand fly mosquitoes Lutzomyia longipalpis and Lutzomyia cruzi, present in several regions of Brazil, including the state of Sergipe, where they are considered endemic. Although few cases have been reported in other states of Brazil, no case of cutaneous leishmaniasis in horses has been reported in Sergipe. The objective of this study was to report a case of cutaneous leishmaniasis in a horse in the municipality of Itaporanga d'Ajuda, Sergipe, Brazil.

Case: A 2-year-old female quarter-horse was examined by a private veterinarian due to the presence of a nodular lesion on the mucocutaneous region of the lips. Macroscopically, the nodule was alopecic with depigmented areas, measuring approximately $1.0 \mathrm{~cm}$ in diameter, and there was a focal ulcer in the region of the labial commissure. Excisional biopsy was performed as follows; the material obtained was fixed in $10 \%$ buffered formalin and sent for histopathological examination. Further, it was subjected to routine histological processing by embedment in paraffin and hematoxylin-eosin staining. Microscopically, there were intense multifocal to coalescent lymphohistioplasmocytic infiltrates with epithelioid macrophages and multinucleated giant cells, both containing amastigote forms comparable with Leishmania spp. Morphological diagnosis was defined as extensive multifocal to coalescent chronic granulomatous dermatitis with intracytoplasmic amastigote forms. Furthermore, for positive controls, immunohistochemical examination was performed using serum from dogs naturally infected with $L$. $(L$.) infantum, which resulted in a strongly positive immunostaining of innumerable intracytoplasmic amastigote structures, confirming infection by Leishmania infantum.

Discussion: Leishmaniasis is a public health problem and is frequently studied. In the biological cycle of the parasite, canids are considered as reservoirs, and humans are considered as accidental hosts. However, in endemic rural areas, several species of mammals can be infected. Microscopic lesions of cutaneous leishmaniasis are compatible with granulomatous dermatitis, characterized by lymphohistioplasmocytic inflammatory infiltrates with epithelioid macrophages containing amastigote forms. Age, breed, and sex are not considered predisposing factors for this disease and their transmission depends on the existence of the vector in the environment. The diagnosis is based on serological, molecular, and immunohistochemical techniques; in the present study, immunohistochemistry was performed for diagnostic confirmation using serum from dogs naturally infected with $L$. (L.) infantum. It was found that this antibody can be successfully applied in the immunohistochemical diagnosis of leishmaniasis in tissues of horses. Histopathological examination including immunohistochemical analysis was essential to confirm the diagnosis. To the best of our knowledge, this is the first report of Leishmania infantum infection in a horse in the state of Sergipe, Brazil. It is essential to carry out epidemiological studies to define control measures to be implemented in the state since it is important for public health.
\end{abstract}

Keywords: Leishmania (Leishmania) infantum, horses, histopathology, immunohistochemistry, granuloma.

Descritores: Leishmania (Leishmania) infantum, cavalo, histopatologia, imunohistoquímica, granuloma. 


\section{INTRODUCTION}

Leishmaniasis is caused by an obligate intracellular protozoan from genus Leishmania. The disease has distinct clinical-epidemiological presentations, depending on the species of protozoan involved [1].

According to the clinical presentation, two different forms can be observed: the cutaneous form or cutaneous leishmaniasis (CL) and visceral form or visceral leishmaniasis (VL). Nearly 1 million human cases of leishmaniasis are reported every year, of which 95\% are concentrated in the Americas Mediterranean, Middle East, and Central Asia [15]. In the New World, the species involved in LC are Leishmania (Viannia) braziliensis and Leishmania (Viannia) amazonensis. Infection by $L$. $(L$.) infantum is related to severe clinical complications related to VL, a potentially fatal disease for humans and dogs. It has been reported that this agent may also cause CL in horses [2]. In Sergipe, leishmaniasis is considered endemic and it affects humans and horses $[8,10]$.

Transmission occurs through the bite of infected sand flies Lutzomyia longipalpis and Lutzomyia cruzi [3]. L. (L.) infantum is the main agent that infects humans and domestic animals; dogs have a greater participation in the chain of transmission of the disease [10]. There are case reports of CL in equines in Brazil $[6,12]$; however, in the state of Sergipe, no such study has been reported. Thus, the objective of this study was to report a case of cutaneous leishmaniasis in a horse in the municipality of Itaporanga d'Ajuda, Sergipe, Brazil.

\section{CASE}

A 2-year-old quarter horse female from the municipality of Itaporanga d'Ajuda, located in the rural area of the state of Sergipe, Brazil was examined by a private veterinarian, due to the presence of a nodular lesion on the mucocutaneous region of the lips. Macroscopically, the nodule was alopecic with depigmented areas, measuring approximately $1.0 \mathrm{~cm}$ in diameter, and there was a focal ulcer in the region of the labial commissure (Figure 1). Excisional biopsy was performed and the material was fixed in $10 \%$ buffered formalin and sent for histopathological examination to a private laboratory in the vicinity LABOVET Diagnostic Center.

The tissue samples were processed by the paraffin impregnation technique and the slides were stained with hematoxylin-eosin (HE). Microscopically, an intense multifocal to coalescent lymphohistioplasmocytic infiltrate was observed with neovascularization, necrosis, epithelioid macrophages, and multinucleated giant cells, both containing numerous intracytoplasmic structures morphologically comparable with Leishmania spp. amastigotes (Figure 2). The morphological diagnosis was established as extensive multifocal to coalescent chronic granulomatous dermatitis with intracytoplasmic amastigote forms. To confirm the diagnosis, the material was submitted for immunohistochemical examination according to the methodology established by literature [12]. As a positive control, a dog tissue sample known to be positive for $L$. (L.) infantum was used; for the negative control, a mouse tissue sample without significant injury was used.

Initially, the slides were deparaffinized and rehydrated. Subsequently, endogenous peroxidase was blocked using PBS enriched with $4 \%$ hydrogen peroxide for $30 \mathrm{~min}$ at room temperature $\left(25^{\circ} \mathrm{C}\right)$. This was followed by the blockage of nonspecific reactions with casein, using $6 \%$ skimmed milk. The slides were incubated for $30 \mathrm{~min}$ at $25^{\circ} \mathrm{C}$, and subsequently incubated with primary antibodies from canine serum naturally infected with $L$. (L.) infantum [12], in a 1:100 dilution, in a humid chamber at $4^{\circ} \mathrm{C}$ overnight. Further, was used a commercial kit secondary antibody conjugated with streptavidin-biotin-peroxidase ${ }^{1}$. The reaction was performed according to the manufacturer's recommendations. Briefly, $1 \mathrm{~mL}$ of diaminobenzidine (DAB) and $0.05 \mathrm{~mL}$ of the chromogen was applied on the tissue and the slides were incubated for $5 \mathrm{~min}$ at $25^{\circ} \mathrm{C}$. For counterstaining, Harris' hematoxylin was used for $3 \mathrm{~s}$, followed by a wash with running water for $10 \mathrm{~min}$, and subsequently the slides were mounted with coverslips.

Microscopic analysis revealed that in the epidermis and superficial to deep dermis, numerous intracytoplasmic amastigote structures in macrophages were strongly positive. This was revealed with DAB, confirming the diagnosis of infection by $L$. (L.) infantum (Figure 2 A \& B).

\section{DISCUSSION}

Leishmaniasis is a public health problem; therefore, it is frequently studied. In endemic areas, whether rural or urban, several mammals can be infected $[1,3]$.The strong relationship between humans and animals as well as urban expansion to endemic regions, 
S.R.F. Gonçalves, M.M.L. Waterloo, M.L.M. Barretto, et al. 2020. First Report of Equine Leishmaniosis in the State of Serfipe, Brazil.

Acta Scientiae Veterinariae. 48(Suppl 1): 562.

is considered an important factor in the dissemination of the agent, as it interferes with the epidemiological triad: host, environment, and agent [5].

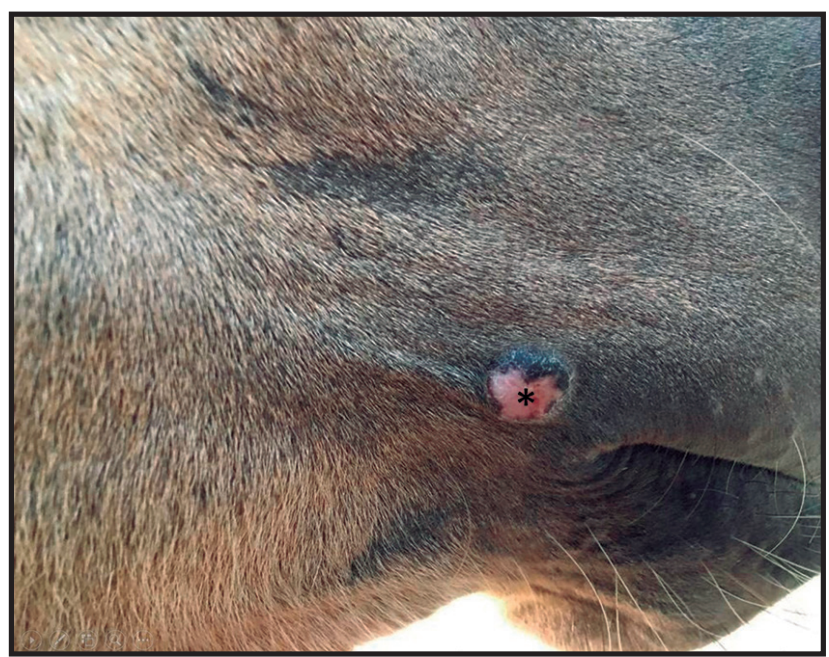

Figure 1. Cutaneous lesion caused by Leishmania infantum in a 2-year-old female quarter horse. A whitish, ulcerated, and alopecic nodule measuring approximately $1.0 \mathrm{~cm}$ in diameter in the mucocutaneous junction region of the lips $(*)$.
For transmission of the disease between susceptible vertebrate hosts, it is necessary to have a vector infected by $L$. (L.) infantum in the promastigote form. The sand fly L. longipalpis is the main vector of the disease and it is abundant in several regions of Brazil, including in Aracaju and its surroundings [8]. The reported animal came from the city of Itaporanga d'Ajuda, located within the surroundings of Aracaju, demonstrating that urban invasion in rural areas and formation of urban conglomerates are key factors for the spread of the vector. Some studies have demonstrated the prevalence of $L$. (L.) infantum in Aracaju in humans and dogs $[7,8,10]$; however, there are no reports on horses. Official state reports of confirmed cases in animals are scarce. It is well known that vector control is the most efficient way to decrease the number of leishmaniasis cases [13], as the existence of the vector in endemic regions is directly related to the onset of the disease. Age, breed, and sex are not considered predisposing factors for the development

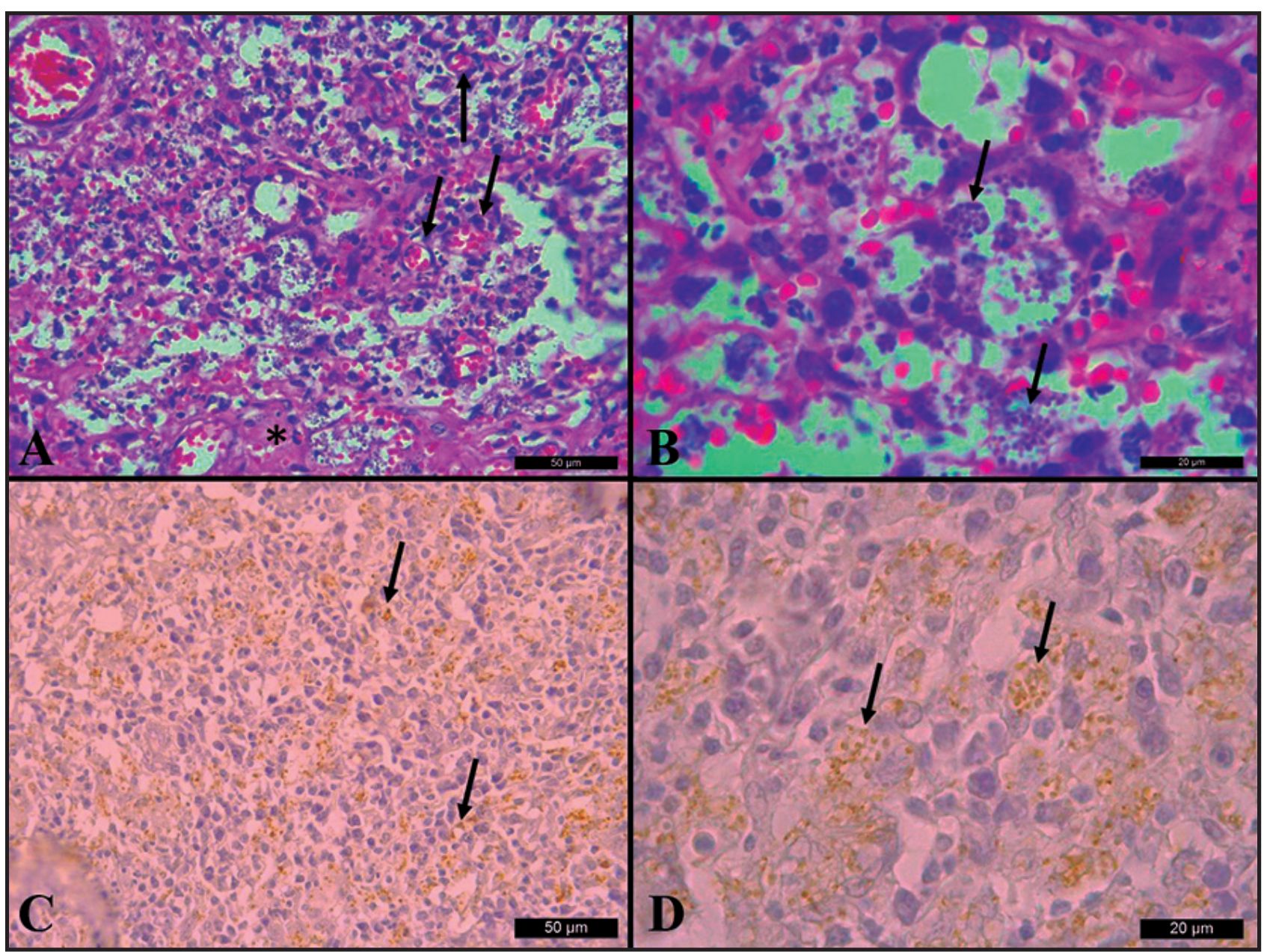

Figure 2. Microscopic analysis of hairy skin in a 2-year-old female quarter horse. A- Intense multifocal to coalescent lymphohistioplasmocytic inflammatory infiltrates with areas of neovascularization (arrow) and necrosis $(*)$ [HE; bar $=50 \mu \mathrm{m}]$. B- Intracytoplasmic amastigote structures in macrophages (arrow) [HE; bar $=20 \mu \mathrm{m}]$. C \& D- Immunohistochemical analysis; amastigotes staining (arrow) with primary antibodies obtained from serum of naturally infected dog with a 1:100 dilution using DAB [Harris' hematoxylin counterstain; bar $=50 \mu \mathrm{m}$ and $20 \mu \mathrm{m}$, respectively]. 
of the disease; in this report, we discuss the case of a 2-year-old female [5,9].

At the time of examination, the animal presented a single nodular lesion in the mucocutaneous region due to the inflammatory response stimulated by $L$. $(L$.) infantum. Macroscopically, the nodule was alopecic, ulcerated with a diameter of approximately $1.0 \mathrm{~cm}$, as reported by literature [9]. The nodular cutaneous presentation is commonly observed in horses, with no reports of other clinical presentations, as normally observed in canids and humans [4].

It is noteworthy that the veterinarian decided to remove the nodule and perform histopathological examination due to an initial suspicion of neoplasia. The importance of complementary examinations for the establishment of differential diagnoses and diagnostic confirmation is emphasized in our study.

The microscopic lesions observed in CL are characterized by the presence of lymphohistioplasmocytic inflammatory infiltrates with epithelioid macrophages containing innumerable amastigote for$\mathrm{ms}$, characterizing a granulomatous reaction $[4,9,11]$, which was also observed in the present study. The formation of a granulomatous lesion is due to the persistence of the infectious agent which proliferates manipulating phagocytosis [14].

The diagnosis is based on serological, molecular, and immunohistochemical techniques $[2,13]$. Immunohistochemistry was essential to confirm the infection. It was marked positive using serum from dogs naturally infected with $L$. (L.) infantum [12]. We conclude that this antibody can be successfully utilized in the immunohistochemical diagnosis of leishmaniasis in the tissues of horses.

Histopathological examination associated with immunohistochemical analysis was essential to determine the diagnosis, which macroscopically could have been incorrectly defined. To the best of our knowledge, this is the first report of $L$. (L.) infantum infection in a horse in the state of Sergipe, Brazil. Considering its importance to public health, it is essential to carry out epidemiological studies to define control measures to be implemented in the state.

\section{MANUFACTURER}

${ }^{1}$ Dako North America Inc. Carpinteria, CA, USA.

Declaration of interest. The authors report no conflicts of interest. The authors alone are responsible for the content and writing of the paper.

\section{REFERENCES}

1 Alvar J., Vélez I.D., Bern C., Herrero, M., Desjeux P., Cano J., Jannin J. \& den Boer M. 2012. Leishmaniasis worldwide and global estimates of its incidence. PLOS ONE. 7(5): 1-12.

2 Benassi J.C., Benevenga G.U., Ferreira H.L., Soares R.M., Silva D.T., Pereira V.F., Ruiz V.L.A. \& Oliveira T.M.F.S. 2018. Molecular and serological detection of Leishmania spp. in horses from na endemic área for canine visceral leishmaniasis in southeastern Brazil. Pesquisa Veterinária Brasileia. 38(6): 1058-1063.

3 Brasil. 2014. Ministério da Saúde. Secretaria de Vigilância em Saúde. Manual de Vigilância e Controle da Leishmaniose Visceral. 5. Brasília: Ministério da Saúde, 120p.

4 Escobar T.A., Dowich G., Santos T.P., Zuravski L., Duarte C.A., Lübeck I. \& Manfredini V. 2019. Assessment of Leishmania infantum infection in equine populations in a canine visceral leishmaniosis transmission area. BMC Veterinary Research. 15(381): 1-9.

5 Feitosa F.L.F., Leal J., Mendes L.C.N., Peiró J.R., Perri S.H.V., Lima V.M.F. \& Marcondes M. 2012. Estudo soroepidemiológico de leishmaniose em equinos na região de Araçatuba-SP, Brasil, área endêmica para leishmaniose visceral. Brazilian Journal of Veterinary Research and Animal Science. 49(6): 500-502.

6 Gama A., Elias J., Ribeiro A.J., Alegria N., Schallig H.D.F.H., Silva F., Santarém N., Cardoso L. \& Cotovio M. 2014. Cutaneous leishmaniosis in a horse from northern Portugal. Veterinary Parasitology. 200(1-2): 189-192.

7 Góes M.A.O., Jeraldo V.L.S. \& Oliveira A.S. 2014. Urbanização da leishmaniose visceral: aspecto clínicos e epidemiológicos em Aracaju, Sergipe, Brasil. Revista Brasileira de Medicina de Família e Comunidade. 9(31): 119-126.

8 Jeraldo V.L.S., Góes M.A.O., Casanova C., Melo C.M., Araújo E.D., Brandão Filho S.P., Cruz D.E.R. \& Pinto M.C. 2012. Sandfly fauna in na área endemic for visceral leishmaniasis in Aracaju, State of Sergipe, Northeast Brazil. Revista da Sociedade Brasileira de Medicina Tropical. 45(3): 318-322.

9 Koehler K., Stechele M., Hetzel U., Domingo M., Schönian G., Zahner H. \& Burkhardt E. 2002. Cutaneous leishmaniosis in a horse in southern Germany caused by Leishmania (Leishmania) infantum. Veterinary Parasitology. 109(1-2): 9-17. 
10 Oliveira C.S., Santos R.D.F.R., Lima L.C.S., Santos R.E.V. \& Almeida A.S. 2019. Perfil epidemiológico de leishmaniose visceral em Aracaju, Sergipe. In: $2^{\circ}$ Congresso Internacional de Enfermagem (Aracaju, Brazil). pp.1-5.

11 Silva F.L., Tafuri W.L., Oliveira M.R. \& Tafuri Wg.L. 2002. Histopathological and immunohistochemical study of the gastrointestinal tract from a dog naturally infected with Leishmania (Leishmania) chagasi: a case report. Arquivo Brasileiro de Medicina Veterinária e Zootecnica. 54(4): 340-344.

12 Tafuri W.L., Santos R.L., Arantes R.M.E., Gonçalves R., Melo M.N., Michalick M.S.M. \& Tafuri W.L. 2004. An alternative immunohistochemical method for detecting Leishmania amastigotes in paraffin-embedded canine tissues. Journal of Immunological Methods. 292(2004): 17-23.

13 Ursine R.L., Dias J.V.L., Morais H.A. \& Pires H.H.R. 2016. Human and canine visceral leishmaniasis in an emerging focus in Araçuaí, Minas Gerais: spatial distribution and socio-environmental factors. Memórias do Instituto Oswaldo Cruz. 111(8): 505-511.

14 Werner P.R. 2010. Inflamação. In: Patologia Geral Veterinária Aplicada. São Paulo: Roca, pp.233-270.

15 World Health Organization. 2010. Control of the leishmaniases - Report of a meeting of the WHO Expert Committee on the Control of Leishmaniases. Geneva, Switzerland. (WHO-Technical Report Series, 949).186p. 